\title{
1110 - Axial-Radial Heterostructures of Telluride Nanowire
}

Debadarshini Samantaray ${ }^{1}$, Abinash Kumar ${ }^{2}$, Priyadarshini Ghosh ${ }^{1}$, Dipanwita Chatterjee ${ }^{1}$, Pavithra Bellare $^{1}$ and N Ravishankar ${ }^{1}$

${ }^{1}$ Indian Institute of Science, Bangalore, Bangalore, Karnataka, India, ${ }^{2}$ Massachusetts Institute of Technology, Cambridge, Massachusetts, United States

Semiconducting nanostructures with modulated composition / crystallography are important for both fundamental understanding as well as technological implications. ${ }^{1-3}$ The properties exhibited by these heterostructures are often superior to their individual counterparts. Moreover, due to increased multi functionality, they find numerous applications in nanoelectronics and photonic. Among these semiconductors, Te (low- band gap semimetal) finds broad application in the field of optoelectronics and thermo-electrics and it's derivatives like lead telluride and bismuth telluride are used as state-of-the-art thermoelectric material. Though it is known that coupling them in 1D could increase the thermoelectric efficiency ${ }^{4}$ (ZT) to a value of 5-6, but a general and simple scheme to achieve the same with coherent interface is still challenging. Here, we demonstrate a simple, scalable and highly reproducible wet chemical synthesis method to achieve tellurium based 1D heterostructure and their formation mechanism, studied using various electron microscopy techniques. ${ }^{5}$ Further, more complex heterostructures of $\mathrm{Bi}_{2} \mathrm{Te}_{3}-$ $\mathrm{PbTe}$ (PBT superlattice nanowire) have been designed using the previous one as template.

Initial Te nanowires, synthesized via microwave method has been used as template to grow Te-PbTe nanowire, where PbTe beads are spaced along the length of the Te nanowire (due to reactive dewetting). The initial Te nanowires have an average diameter of $60 \mathrm{~nm}$ with length in the order of 5-10 micron. HRTEM images of these nanowires reveal that these are single crystalline in nature and [0001] of Te is parallel to [111] of PbTe. The 3-D structure and composition of these beaded nanowires have been investigated using STEM-EDXS tomography. Z-contrast of orthoslices confirms presence of Te throughout the core and PbTe on the beads and volume rendering confirms the cubic shape of PbTe.

These beaded Te-PbTe nanowires have been converted to $\mathrm{PbTe}-\mathrm{Bi}_{2} \mathrm{Te} 3$ superlattice (SL) nanowires (fig:1a,b) by reacting the exposed part of Te with Bi-precursor. Elemental analysis confirms the presence of $\mathrm{Pb}$ only at the cube region where as Te is present throughout the wire and $\mathrm{Bi}$ along the shell part of the wire (fig:1c). Atomic resolution HAADF - STEM (High Angle Annular Dark Field- Scanning Transmission Electron Microscopy) image (fig:2) from the junction of wire and cube depicts a clear interface between the cube and wire and a core shell geometry of $\mathrm{Te}_{-} \mathrm{Bi}_{2} \mathrm{Te}_{3}$ in the wire region. To find out the zone axis relationship between them, PED (precession electron diffraction) studies have been carried out on several such SL nanowires.

These SL nanowires have been found to retain their single crystalline nature and local electron transport measurement on them depict rectifying behavior. To understand the origin of the rectifying nature, various microscopic techniques like EFM (Electrostatic Force Microscopy), KPFM (kelvin Probe Force Microscopy)has been employed. This system can be used as an ideal candidate to study electron transport through semiconducting nanojunction using various insitu-electron microscopic technique. Since the junction between $\mathrm{PbTe}-\mathrm{Bi}_{2} \mathrm{Te}_{3}$ is type-II heterostructure, this could be interesting for various optoelectronic application. Further, SL nanowires with varying composition has been synthesized to find an optimum superlattice nanowire structure for thermoelectric application. 

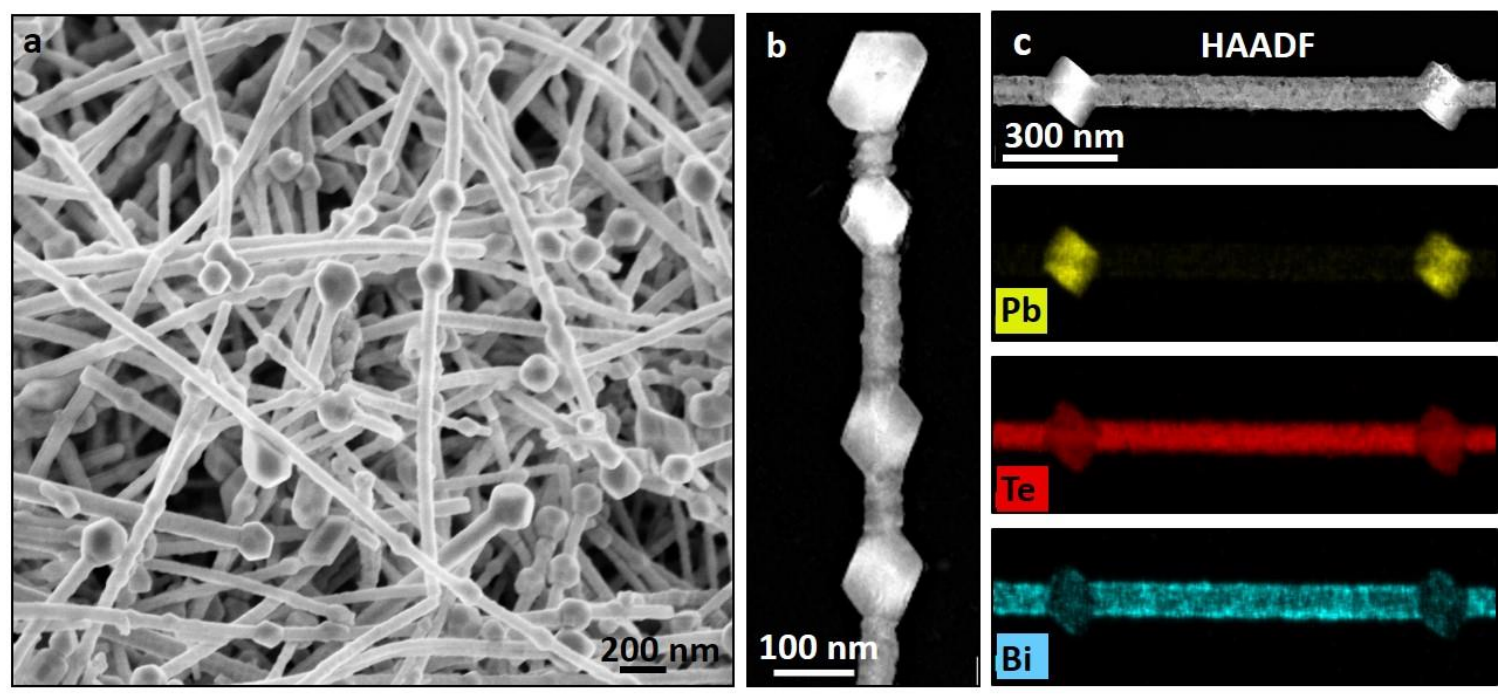

Figure 1. (a) represents low magnification SEM micrograph of PbTe-Bi2Te3 superlattice nanowire, (b) HAADF-STEM image of the same,(c) depicts HAADF-STEM-EDXS mapping from one such nanowire (as evident from the image, the core is Te rich whereas the cube has $\mathrm{Pb}$ and the shell part of wire is $\mathrm{Bi}$ rich).
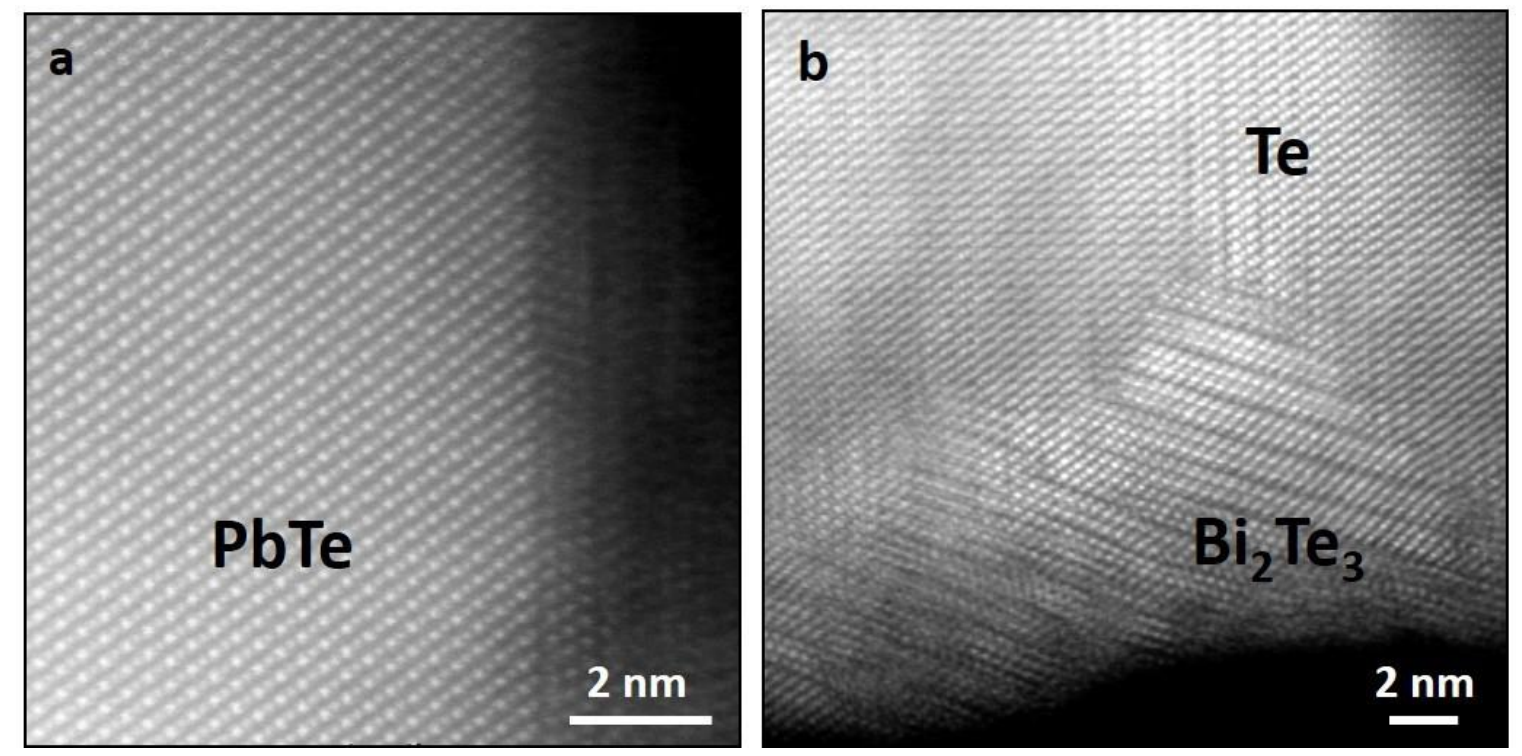

Figure 2. represent high magnification STEM-HAADf image of (a) PbTe cube, (b)Te-Bi2Te3 core-shell structure from the wire region.

References

(1) Kundu, P.; Anumol, E. A.; Nethravathi, C.; Ravishankar, N. Existing and Emerging Strategies for the Synthesis of Nanoscale Heterostructures. 2011, 19256-19269.

(2) Kundu, S.; Kundu, P.; Tendeloo, G. Van; Ravishankar, N. Au 2 S x / CdS Nanorods by Cation Exchange: Mechanistic Insights into the Competition Between Cation-Exchange and Metal Ion Reduction. 2014, 3895-3900.

(3) Jain, P. K.; Amirav, L.; Aloni, S.; Alivisatos, A. P. Nanoheterostructure Cation Exchange : Anionic Framework Conservation. J. Am. Chem. Soc. 2010, 132, 9997-9999. 
(4) Lin, Y. M.; Dresselhaus, S. Thermoelectric Properties of Superlattice Nanowires. Phys. Rev. B Condens. Matter Mater. Phys. 2003, 68 (7), 1-14.

(5) Kumar, A.; Kundu, S.; Samantaray, D.; Kundu, P.; Zanaga, D.; Bals, S.; Ravishankar, N. Designing Diameter-Modulated Heterostructure Nanowires of PbTe/Te by Controlled Dewetting. Nano Lett. 2017, 17 